\title{
La elección de 2017 y el fraccionamiento del sistema de partidos en Chile
}

\author{
The 2017 elections and the fragmentation of the party system in Chile
}

\author{
KeNNETH BUNKER ${ }^{1}$ \\ Universidad Sapienza de Roma, Italia.
}

\begin{abstract}
RESUMEN El propósito de este artículo es reportar los resultados de la elección presidencial y legislativa de Chile en 2017 y explorar su efecto sobre el sistema de partidos. Relata el contexto, y sugiere que hubo tres temas recurrentes que definieron tanto la nominación de los candidatos como el tono de la campaña: una importante polarización política, dos grandes escándalos de corrupción y el debut de un nuevo sistema electoral. Presenta a los candidatos presidenciales y sus principales bases de apoyo. Describe los puntos medulares de la elección y las principales estrategias electorales. Repasa los resultados de la elección, los analiza y los interpreta. Destaca el inédito fraccionamiento en el sistema de partidos provocado por el contexto político e institucional de los últimos años. Sugiere que a partir de 2018 el sistema de partidos está en uno de sus niveles más fragmentados de su historia. Especula que hacia delante los próximos gobiernos serán más flexibles que los anteriores y buscarán pasar legislación sobre la base de negociaciones y pactos multilaterales.
\end{abstract}

PALABRAS CLAVE Elección presidencial, elección legislativa, reforma electoral, sistema de partidos, sistema electoral.

ABSTRACT The purpose of this article is to report the results of Chile's presidential and legislative elections in 2017 and explore their effect on the party system. It relates the context and suggests that there were three recurring

1. PhD. en Ciencia Política. Profesor visitante Universidad Sapienza de Roma. kabunker@ gmail. com 
themes that defined both the nomination of the candidates and the tone of the campaign: strong political polarization, two major corruption scandals, and the debut of a new electoral system. The article presents the presidential candidates and their main support bases and describes the central points of the election and the main electoral strategies. It reviews, analyzes and interprets the results of the election, highlighting the unprecedented fragmentation in the party system caused by the political and institutional context of recent years. We suggest that as of 2018 the level of fragmentation in the party system is among the highest in its history. We speculate that henceforth governments will be more flexible than in the past and will seek to pass legislation based on multilateral agreements and negotiation.

KEYWORDS Presidential election, legislative election, electoral reform, party system, electoral system.

\section{Introducción}

El 19 de noviembre de 2017 los chilenos acudieron a las urnas para votar en la séptima elección presidencial desde el retorno de la democracia. Como en la mayoría de las ocasiones anteriores, la elección fue un referéndum sobre el gobierno saliente ${ }^{2}$. Mientras que algunos votantes se presentaron para sufragar a favor de la continuidad de la obra del segundo gobierno de la presidenta socialista Michelle Bachelet, otros lo hicieron para votar en contra. Ocho candidatos se inscribieron para competir.

Dado que ninguno logró obtener una mayoría absoluta en la primera vuelta, las dos candidaturas con mayorías relativas pasaron a una segunda ronda a disputarse un mes después. El 17 de diciembre de 2017 se confrontaron el candidato de la continuidad, el senador de centroizquierda Alejandro Guillier y el candidato de la alternancia, el expresidente de centroderecha Sebastián Piñera. El segundo ganó con 56,4\% de los votos, y fue elegido presidente de Chile para el periodo 2018-2022.

La elección presidencial, que se celebró junto con la elección legislativa, se dio en medio de un clima de relativa inquietud e incertidumbre. Como se relata en la siguiente sección, una inusitada polarización ideológica y dos grandes escándalos de corrupción generaron inquietud sobre cómo reaccionaría la ciudadanía en las urnas. A su vez, la aplicación del nuevo conjunto de reglas electorales para elegir a senadores y diputados provocó incertidumbre sobre cómo los votos se transformarían en escaños.

Este artículo recuenta los hechos que se dieron alrededor de la elección presidencial y legislativa de 2017 para explorar si hubo una transmutación importante en 
el sistema de partidos. Para determinar aquello, no solo es importante describir los principales eventos que sucedieron en la antesala de la elección, con especial enfoque en la carrera presidencial, sino que, también, es crucial mirar los resultados de la elección legislativa. Es de esperar que la elección presidencial impacte en la elección legislativa ${ }^{3}$, y que ambas en conjunto impacten en el sistema de partidos ${ }^{4}$.

El contexto de la elección presidencial es importante para entender las estrategias de los partidos para enfrentar la elección legislativa. Por eso, este artículo comienza allí. Un recuento descriptivo relativo a los candidatos presidenciales y sus bases de apoyo no solo es útil para entender por qué los partidos se ordenaron tácticamente en la forma en que lo hicieron, sino que, además, para entender cómo influyeron para causar potenciales efectos de largo plazo. Es imposible entender el sistema de partidos sin las observar las dinámicas que se dan en elecciones presidenciales ${ }^{5}$.

Lo que resta de este artículo se estructura de la siguiente forma. La segunda sección relata el contexto de la elección. La tercera presenta a los candidatos presidenciales, sus principales bases de apoyo y describe cómo reaccionaron frente a algunos de los puntos medulares. La cuarta analiza los resultados y destaca el fraccionamiento en el sistema de partidos y el recambio en el Poder Legislativo. La sección final resume el escenario político resultante, y enfatiza en la adopción de un modo de gobernar basado en negociaciones y pactos multilaterales.

\section{El contexto de la elección de 2017}

Parte de la razón de la derrota del oficialismo tiene que ver con la creciente polarización política que alcanzó su peak durante el cuatrienio de Michelle Bachelet (2014$2018)^{6}$. La tendencia en esa dirección se acentuó con las revisiones constitucionales propuestas por el gobierno. La profundidad y el simbolismo ideológico tras las reformas los obligó a los chilenos a escoger entre apoyar la vía progresista del cambio estructural, representado por Michelle Bachelet y su gobierno, y el camino moderado de la responsabilidad fiscal, propuesto por Sebastián Piñera y la oposición?

La fuerza con que Michelle Bachelet buscó aprobar las reformas fue fundamental para generar este clima. Luego de su instalación en 2014, el gobierno hizo todo lo posible para empujar su agenda legislativa. De hecho, tuvo bastante éxito solo en el primer año de gobierno, en el cual logró aprobar las reformas tributaria, educacional y electoral $^{8}$. Y a pesar de no poder cumplir con la todas sus promesas de campaña,

\section{JONES (1999).}

4. NETO y COX (1997).

5. SHUGART Y TAAGEPERA (2017).

6. Véanse ALEMÁN (2008); FÁBREGA et al. (2018); LONDREGAN (2000).

7. La Nación (2017).

8. ARANA (2016). 
pudo avanzar bastante, como en la de una Nueva Constitución, que en su etapa de diseño involucró a más de doscientas mil personas9

Como respuesta a esta agenda reformista, la oposición intentó llevar el debate hacia el coste económico. Sebastián Piñera y la coalición de derecha buscaron advertir que las reformas no solo eran caprichosas y estaban mal diseñadas, sino que, además, implicaban costos inconmensurables ${ }^{10}$. El modus operandi de la ofensiva se pudo observar con mayor claridad en los ataques a la reforma tributaria, donde la oposición fue tajante en acusar al gobierno de Michelle Bachelet de dogmatismo e improvisación ${ }^{11}$.

Por su parte, el oficialismo enfrentó serios problemas políticos para hacerle frente a las críticas. A pesar de pasar tres grandes reformas estructurales en el comienzo de su periodo, al poco andar se vio obligado a hacer grandes cambios en el gabinete ${ }^{12}$. La salida de todo el círculo cercano a la Presidenta dentro de los dos primeros años del mandato obligó al gobierno a pasar de una táctica proactiva a una estrategia defensiva. Si bien la administración buscó pasar reformas hasta el final de su cuatrienio, su éxito fue limitado ${ }^{13}$.

Este y otros debates ideológicos y políticos que se dieron durante el cuatrienio de Michelle Bachelet estuvieron marcados por dos grandes escándalos de corrupción. Uno fue el caso Caval, en el que se vio envuelto el propio hijo de Michelle Bachelet (Sebastián Dávalos), y el otro fue el caso SQM, en el cual se vieron involucrados senadores y diputados de todos los partidos. Mientras que, primero, tuvo un efecto sobre la popularidad de la Presidenta, el segundo tuvo un costo transversal sobre la legitimidad de toda la clase política.

Poco tiempo después de darse a conocer los hechos relacionados al caso Caval (en febrero de 2015), los índices de aprobación presidencial se desplomaron. En agosto de 2016, la encuesta Adimark mostró a la gobernante en 15\% de aprobación, el índice más bajo para cualquier Mandatario desde el retorno de la democracia ${ }^{14}$. A su vez, en marzo de 2017, la encuesta CEP mostró una caída similar para varios de los líderes políticos tradicionales ${ }^{15}$. El castigo afectó a todos, desde los alcaldes más desconocidos hasta los presidentes de partido menos influyentes.

\footnotetext{
9. NAVIA (2018).

10. GAMBOA y SEGOVIA (2016).

11. El Mercurio (2017).

12. TELETRECE (2017).

13. FUENTES (2018).

14. Véase encuesta ADIMARK (2016).

15. Véase encuesta Cep (2017).
} 
Este escenario tuvo un impacto significativo sobre la nominación de candidatos ${ }^{16}$. Por ejemplo, varios senadores titulares no pudieron ir por la reelección por estar inmiscuidos en procedimientos legales (como el senador Jaime Orpis). Algunos, pudiendo optar por la reelección, decidieron no hacerlo por sus sendas caídas en índices de aprobación (como el senador Patricio Walker). Otros, a pesar de haber estado directamente involucrados en las irregularidades, decidieron repostular de igual manera (como el senador Fulvio Rossi).

En la misma línea, es imposible ignorar el efecto que tuvo la anticipación del debut del nuevo sistema electoral sobre algunas de las definiciones más importantes ${ }^{17}$. Ante los nuevos incentivos institucionales, los partidos se vieron obligados a rearticular sus estrategias políticas y redefinir sus modos de afrontar la competencia. El aumento en el número total de legisladores a elegirse, así como el incremento en el número de escaños disponibles en cada uno de los distritos, obligó a los partidos a cambiar sus tácticas.

Normalmente, la modificación a uno de los elementos más importantes de la estructura electoral (magnitud de distrito) conlleva a un cambio en los modos de coordinación entre los distintos partidos ${ }^{18}$. Pero más importante, la evidencia sugiere que cambios en los sistemas electorales llevan a cambios en los sistemas de partidos ${ }^{19}$. Este es, al menos, el caso en Chile ${ }^{20}$. Todas las reformas electorales han impactado tanto la coordinación de los partidos antes de la elección como el sistema de partidos después de la elección.

La combinación entre el clima político polarizado, el escenario de baja popularidad política y la inminente aplicación del nuevo sistema electoral, inevitablemente sugirió que habría una transmutación importante en el sistema de partidos. Algunos pronosticaron que el sistema volvería a ser uno de tres tercios, similar al existente previo al quiebre de la democracia en 1973, mientras que otros auguraron que resultaría un nuevo ordenamiento multipartidario, sin grandes coaliciones o partidos dominantes ${ }^{21}$.

Asimismo, se especuló sobre las características que tendría el nuevo Congreso que, para algunos, estaría marcado por un recambio inédito. Esta idea no solo estuvo relacionada a la potencial entrada de varios partidos nuevos a la competencia, sino, también, a la noción de que el potencial castigo electoral sería notoriamente fuerte

16. BUNKER (2017).

17. Véase GAMBOA y MORALES (2015).

18. RAE (1967).

19. COX (1997).

20. NAVIA (2005).

21. COOPERATIVA (2017). 
para los partidos y candidatos tradicionales. Como se mencionó atrás, varios de los legisladores titulares, tanto senadores como diputados, no buscarían la reelección o, bien, se especulaba que los que si lo hacían sería con poco éxito ${ }^{22}$.

\section{Candidatos presidenciales, coaliciones y partidos políticos}

Ocho candidatos presidenciales se inscribieron en el Servicio Electoral de Chile (Servel) para competir en la elección de 2017. Los primeros en hacerlo fueron quienes participaron, y ganaron, en las primarias presidenciales del 2 de julio del mismo año (véase tabla 1). Las primarias, reguladas por la Ley electoral, proveyeron que cada coalición pudiese presentar todos los candidatos que quisieran para definir a su representante común, con la salvedad de que los resultados fueran vinculantes, y que el ganador quedara automáticamente inscrito.

Uno de ellos fue el expresidente Sebastián Piñera, que ganó las primarias de la coalición de derecha (Chile Vamos), al vencer al senador Manuel José Ossandón y al diputado Felipe Kast. La victoria le permitió inscribir su tercera candidatura presidencial (su primera campaña fue en 2005, cuando perdió ante Michelle Bachelet, y su segunda fue en 2009, cuando le ganó al entonces expresidente Eduardo Frei RuizTagle). Al igual que en sus incursiones previas, se buscó presentar como el candidato de la bonanza económica.

Beatriz Sánchez también inscribió su candidatura tras ganar en las primarias de su coalición (Frente Amplio). Luego de una breve, pero intensa campaña, logró derrotar a Alberto Mayol, para transformarse en la nominada oficial. Periodista de profesión, buscó representar a los votantes que en elecciones anteriores habían apoyado a los candidatos y partidos de la Concertación (entre 1989 y 2012) y Nueva Mayoría (desde 2013). Aprovechando el desprestigio de la política tradicional, se buscó presentar como una outsider independiente.

22. BUNKER (2017). 
Tabla 1. Resultados de las elecciones presidenciales primarias de 2017.

\begin{tabular}{|l|c|c|}
\hline & Número de votos & Porcentaje de votos \\
\hline Chile Vamos & & \\
\hline Sebastián Piñera & 827.434 & 58,4 \\
\hline Manuel José Ossandón & 372.215 & 26,2 \\
\hline Felipe Kast & 218.489 & 15,4 \\
\hline Total* $^{*}$ & $\mathbf{1 . 4 1 8 . 1 3 8}$ & $\mathbf{1 0 0 , 0}$ \\
\hline
\end{tabular}

\begin{tabular}{|l|c|c|}
\hline Frente Amplio & & \\
\hline Beatriz Sánchez & 221.416 & 67,6 \\
\hline Alberto Mayol & 106.300 & 32,4 \\
\hline Total* & $\mathbf{3 2 7 . 7 1 6}$ & $\mathbf{1 0 0 , 0}$ \\
\hline
\end{tabular}

Fuente: Servicio Electoral de Chile."Solo suma el total de votos válidos; no suma votos nulos ni blancos.

Los otros seis candidatos se inscribieron directamente en el Servel. Algunos lo hicieron tras ser apoyados por un partido político, mientras que otros lo hicieron presentando las treinta mil firmas requeridas. Eduardo Artés siguió la primera ruta, inscribiendo su candidatura el 22 de julio, con el respaldo del partido Unión Patriótica (UPA). Buscó representar el nicho de votantes de izquierda abandonados por el Partido Comunista. Usando a Corea del Norte y Venezuela como modelos, se buscó presentar como el único candidato genuinamente de izquierda ${ }^{23}$.

El cuarto en inscribirse fue Alejandro Guillier, el 4 de agosto. A pesar de ser independiente, fue apoyado por la coalición de centroizquierda, La Fuerza de Mayoría. Como sucesor de la presidenta Michelle Bachelet, buscó representar un proyecto de continuidad con el gobierno saliente, pero con algunos visos de moderación. Elegido senador para el periodo 2014-2022, se presentó como un socialdemócrata a medio camino entre la Concertación y la Nueva Mayoría. Su coalición lo buscó presentar como un candidato progresista responsable.

Su campaña llegó para mostrarse como un contraste a la campaña de Beatriz Sánchez. Dado que ambos candidatos provenían del mundo de los medios de comunicación e, incluso, trabajaron juntos en el pasado, la diferenciación implicaba un trabajo estratégico mayor. En esa línea, el senador buscó mostrar su apoyo de una coalición tradicional como una de sus principales ventajas frente a Beatriz Sánchez, que, por su parte, era apoyada por una coalición nueva que contaba una representación política significativamente pequeña.

23. BIOBÍO (2017). 
José Antonio Kast y Alejandro Navarro inscribieron sus candidaturas el mismo día, el 18 de agosto. Mientras que el primero se registró luego de juntar las firmas solicitadas por el Servel, el segundo lo hizo con el apoyo del partido PAIS. Ambos representaron polos opuestos. José Antonio, como exmilitante de la Unión Demócrata Independiente, buscó representar a un electorado de extrema derecha, mientras que Alejandro, un exmilitante del Partido Socialista, buscó representar a un electorado de extrema izquierda.

Carolina Goic inscribió su candidatura el 19 de agosto y Marco Enríquez Ominami el 20 de agosto. Carolina fue la cuarta candidata de la Democracia Cristiana (DC) en competir en una elección presidencial desde 1990, pero la primera en competir por fuera de una gran coalición desde 1970. A su vez, Marco fue por su tercera aventura consecutiva, luego de perder en las primeras vueltas de 2009 y 2013. Ambos candidatos, apelaron al voto blando de la centroderecha y centroizquierda, respectivamente.

En paralelo a los candidatos presidenciales, nueve coaliciones se inscribieron para competir en la elección legislativa. Cada una de estas coaliciones, compuestas por múltiples partidos políticos, presentó una lista con candidatos a senador (en siete circunscripciones) y diputado (en veintiocho distritos). Algunas coaliciones se enfocaron en competir en algunas localidades más que otras. En total, 196 candidatos compitieron por veintitrés escaños disponibles en el Senado y 960 candidatos compitieron por 155 escaños disponibles en la Cámara de Diputados.

La coalición que respaldó a Sebastián Piñera (Chile Vamos) estuvo compuesta por cuatro partidos: la Unión Demócrata Independiente (UDI), Renovación Nacional (RN), Evolución Política (Evopoli) y el Partido de Regionalistas Independientes (PRI). Los dos primeros partidos son los fundadores de la alianza de derecha y han competido juntos en todas las elecciones presidenciales y legislativas desde 1989. A su vez, fue la primera de Evopoli y el PRI en la coalición. De hecho, fue la primera de Evopoli en cualquier elección.

La coalición de Alejandro Guillier (La Fuerza de la Mayoría) enfrentó la elección con menos partidos que en la anterior. Mientras que se mantuvieron fijos el Partidos Socialista (PS), el Partido Por la Democracia (PPD) y el Partido Radical (PR), el Partido Comunista (PC) reemplazó a la Democracia Cristiana (DC) en la alineación titular. Si bien el PC formó parte de la coalición para la elección de 2013, y presentó algunos candidatos al Congreso en esa ocasión, no tuvo mucha incidencia en las decisiones estratégicas.

A su vez, la DC presentó su propia alternativa (llamada Convergencia Democrática) para respaldar a Carolian Goic y la larga lista de candidatos a senador y diputado. La DC fue acompañada en la coalición por dos partidos pequeños, el MAS y la Izquierda Ciudadana (IC). La decisión de abandonar la coalición en que participaron desde 1989 se debió a una serie de disputas con el PC por espacios de poder y linea- 
mientos ideológicos. La entrada del PC a la coalición implicó una caída en la capacidad del partido de ejercer poder dentro de la coalición.

Pero la decisión de la DC, de abandonar la coalición, también se debió a que algunos académicos ligados al partido encontraron que bajo el nuevo sistema electoral (proporcional) el partido tendría menos probabilidades de sufrir una derrota que bajo el anterior (binominal) ${ }^{24}$. Argumentaron que la caída en la barrera de entrada y su lista nacional los favorecería en la traducción de votos en escaños. Otros académicos, mediante simulaciones electorales, encontraron que si el partido abandonaba la coalición sufriría su peor derrota histórica ${ }^{25}$.

La conglomeración de partidos que apoyó a Beatriz Sánchez (Frente Amplio) estuvo compuesta por seis partidos, encabezados por Revolución Democrática (RD), el Partido Humanista (PH) y el Partido Liberal (PL). Esos tres partidos contaban con los únicos diputados titulares de la coalición: Giorgio Jackson, Gabriel Boric y Vlado Mirosevic, respectivamente. Es importante notar que además de estos partidos, hubo una serie de otros movimientos que también participaron apoyando a Beatriz en la carrera presidencial y presentaron candidatos a la elección legislativa.

Por su parte, los candidatos presidenciales Marco Enríquez-Ominami y Alejandro Navarro fueron endosados por la misma coalición (Por Todo Chile). Si bien compitieron de forma independiente en la elección presidencial, colaboraron en confeccionar la lista legislativa. A su vez, Eduardo Artés fue apoyado por su propia coalición (Unión Patriótica). Además, hubo otras tres listas legislativas que levantaron candidatos a senador y diputado, pero que no apoyaron a ningún candidato presidencial en particular: la Coalición Regionalista Verde, Sumemos y el Partido de Trabajadores Revolucionarios.

\section{La campaña electoral, las estrategias y los debates}

La campaña electoral comenzó noventa días antes de la elección presidencial. Un tema recurrente fue las evaluaciones y juicios al gobierno saliente. Al igual que los votantes, los candidatos se dividieron. Mientras que los dos de derecha coincidieron en el rechazo a la continuidad, los seis candidatos de izquierda se dividieron. Marco Enríquez-Ominami, Carolina Goic, Alejandro Guillier y Beatriz Sánchez plantearon la necesidad de una continuidad moderada, mientras que Eduardo Artés y Alejandro Navarro postularon la urgencia de una profundización radical.

Otro tema recurrente a lo largo de la campaña giró en torno a los escándalos de corrupción. Para los candidatos endosados por los partidos tradicionales fue una cuestión particularmente sensible. Por un lado, se vieron obligados a condenar las

24. MIHOVILOVIC y MORAGA (2017).

25. BUNKER (2017); MORALES (2017). 
irregularidades, pero, por otro, tuvieron que ser cuidadosos de no ser víctimas colaterales de sus propias críticas. Algunos candidatos, incluso, tuvieron que enfrentar interpelaciones directas, como fue el caso de Marco Enríquez-Ominami, que en el transcurso de la campaña fue involucrado en las irregularidades por la Fiscalía Nacional $^{26}$.

Un tercer tema giró en torno a los potenciales efectos de la reforma electoral. A pesar de no influir directamente en el debate ideológico, formó parte importante de la conversación política. La proliferación de alternativas obligó a los candidatos a modificar sus estrategias. Esto fue notoriamente diferente a elecciones anteriores, que se disputaban entre dos bandos. El simple hecho de tener ocho candidatos presidenciales y nueve listas legislativas influyó tanto en la relación entre candidatos como en el modo de interacción entre candidatos y votantes.

En referencia al rol Bachelet en la elección, es importante volver a destacar que en términos ideológicos solo dos de los seis candidatos se identificaron con la derecha mientras que el resto lo hizo con el centro o la izquierda. Esta distribución les daba una ventaja importante a los candidatos de derecha, que en caso de pasar alguno de ellos a segunda vuelta, tendrían una base electoral más cohesionada. Esta heurística electoral fue funcional para que Sebastián Piñera y José Antonio Kast diseñaran campañas implícitamente complementarias.

Por su parte, Sebastián Piñera apuntó sus críticas al costo económico de las reformas. Según el ex Presidente, los problemas tras el diseño y la implementación de los cambios estructurales no eran casos aislados, sino que eran frecuentes y característicos del modo de gobernar de la coalición de gobierno. Un ejemplo recurrente usado por el candidato de Chile Vamos fue la redundancia y futilidad del proceso de reforma constitucional. Según él, la presidenta Michelle Bachelet ocupó demasiado tiempo y recursos en una causa perdida.

En contraste, José A. Kast dirigió sus críticas al legado social y cultural de Michelle Bachelet. Buscó rechazar la agenda valórica del gobierno, por medio de críticas a iniciativas relacionadas al aborto y el matrimonio homosexual. A diferencia del enfoque liberal y económico de la campaña de Sebastián Piñera, José Antonio Kast buscó proponer un modelo más tradicional y conservador. En este sentido, ambos hicieron campañas complementarias. Mientras que uno atacó las externalidades económicas, el otro criticó las externalidades valóricas.

Eduardo Artés y Alejandro Navarro también plantearon posturas críticas, pero en una dirección contraria. Ambos sostuvieron que, si bien las reformas de Michelle Bachelet iban en la dirección correcta, eran insuficientes. Esto fue particularmente cierto en el caso de Eduardo Artés, que en más de una ocasión trató al gobierno de

26. La Tercera (2017) 
neoliberal. En cualquier caso, ambos candidatos propusieron darle un giro a la izquierda importante al país. Para ellos, el gobierno, a pesar de ser el más progresista desde el retorno de la democracia, no hizo todo lo que pudo haber hecho.

Beatriz Sánchez también presentó una visión crítica al gobierno, pero buscó matizarlo un poco más, reconociendo que el gobierno había avanzado bastante en temas de equidad social. Sin embargo, considerando que el objetivo principal del Frente Amplio es reemplazar a la centroizquierda, y no renovarla, tuvo que tomar posturas más moderadas para no alienar a los votantes. Por esta misma razón tuvo que proponer un programa económico menos radical de lo que hubiese sido consistente con la ideología de su coalición.

Marco Enríquez-Ominami siguió en una línea similar, pero con menos fuerza, pues no contaba con suficiente capital político para generar masa crítica de apoyo a sus propuestas. Su involucramiento en el caso de SQM le jugó en contra. Al ser asociado con escándalos de corrupción, muchos de sus potenciales votantes migraron hacia la candidatura de Alejandro Guillier o de Beatriz Sánchez, que eran ideológicamente afines, pero sin los flancos judiciales. Su incapacidad de hacer críticas a las irregularidades sindicadas por la Fiscalía Nacional le jugaron en contra.

Por su parte, el candidato oficial de la continuidad, Alejandro Guillier, buscó defender la obra de Michelle Bachelet, pero con mayor templanza. Rara vez sugirió ahondar en las reformas. Más bien, prefirió usar un tono conciliador, que lograra comunicar su aprobación a la buena intención de las revisiones estructurales, pero aceptar que también había espacio para mejorar. Su modelo de campaña fue similar al de la otrora Concertación, pero con un tinte progresista marcado por la reciente incorporación definitiva del Partido Comunista a la coalición.

En comparación al resto de las candidaturas, la de Carolina Goic fue la más moderada. Precisamente porque el objetivo de la DC era apuntar a un público más moderado y de centroderecha, su plataforma de campaña se basó en críticas a la gestión del gobierno. Su principal crítica fue que, si bien la administración había comenzado bien, finalmente había sido cooptado por fuerzas de izquierda. A su vez, trató de reivindicar el papel de un partido capaz de articular política desde el centro, de acorde con los valores tradicionales de los chilenos.

En forma paralela al debate ideológico, las coaliciones y los partidos entraron en importantes conversaciones internas en anticipación de lo que podrían pasar en la transformación de votos en escaños, que por primera vez sería regulado por el nuevo sistema electoral $^{27}$. Tuvieron que lidiar con la sensación generalizada de que habría una fragmentación en el sistema de partidos. Eso implicaba que, por un lado, nacería una serie de partidos políticos nuevos y que, por otro lado, morirían algunos de los acuerdos partidarios vigentes.

27. TORO y VALENZUELA (2018). 
Uno de los factores que se mencionó como factor explicativo fundamental de la potencial fragmentación, fue el aumento en el número de escaños disponibles por cada unidad electoral. Al pasar del binominal al proporcional se pasaba de un sistema que repartía dos escaños en todas las unidades electorales a un sistema que repartía entre tres y ocho escaños dependiendo de la población. Es efecto, mientras que en 1989-2013 era necesario alcanzar un 33\% para asegurar un escaño, en 2017 no sería necesario alcanzar más de $25 \%$ para asegurar lo mismo.

Para agrupaciones políticas menores esto implicaba una gran oportunidad para constituirse como partido. Varios de los movimientos que en elecciones anteriores anticipaban perder en todas las circunscripciones antes las grandes coaliciones, al no poder alcanzar los umbrales mínimos, ahora tenían la esperanza de hacerlo. Fue el caso de algunos partidos como Revolución Democrática por la izquierda y de Evolución Política por la derecha. Tan pronto como se anunciaron las nuevas reglas electorales se transformaron en partidos, y al poco andar en máquinas electorales.

Esto influyó sobre la elección al fragmentar y pluralizar un debate que tradicionalmente se daba entre dos bandos. El caso de Revolución Democrática es ejemplar. Al bajar las barreras de entrada, aumentaron sus incentivos para competir en todas las circunscripciones y distritos. Y, al mismo tiempo, al llevar una lista legislativa, aumentaron sus incentivos para inscribir a una candidata presidencial propia. Al estar en ambas elecciones, aumentó su participación en el debate político nacional y, con eso, su influencia en el ámbito nacional.

Ahora bien, tal como se generaron incentivos para que nacieran partidos políticos nuevos, también se generaron para que murieran más de alguno de los vigentes. En esta línea, un caso emblemático fue el de la Democracia Cristiana. No dejó indiferente a nadie cuando el partido que apoyó a Carolina Goic anunció que perseguiría una opción electoral propia. Como se mencionó más atrás, si bien su definición obedeció fundamentalmente a razones ideológicas, solo se volvió política y electoralmente viable tras la reforma.

La difícil relación de la DC con el resto de los partidos de la coalición de centroizquierda sirvió como antecedente para contextualizar la salida, pero fueron los incentivos de las nuevas reglas electorales las que la justificaron estratégicamente. Si bien siempre hubo un debate sobre un potencial éxodo de la coalición, es casi seguro que bajo el binominal el partido habría sufrido una gran derrota electoral. En cambio, el proporcional moderado ofrecía mejores garantías para que varios de los titulares consiguieran la reelección.

La salida de la DC, y su constitución como una nueva alternativa política, generó tensión en el sector de centroizquierda por la duración de la campaña. Una razón de aquello fue porque al buscar la opción propia se convertían en oposición a un gobierno del cual aún formaban parte. La DC tuvo ministros en el gabinete de Michelle 
Bachelet hasta el último día. Pero también generó tensiones porque tuvo que rivalizar con La Fuerza de la Mayoría en el ámbito nacional en las elecciones legislativas. Partidos que fueron aliados por décadas de pronto se convirtieron en rivales.

La figura 1 muestra las encuestas de intención de voto presidencial para los cuatro candidatos más populares, desde agosto de 2016 hasta noviembre de 2017. La tendencia sugiere que Sebastián Piñera siempre estuvo liderando la carrera, pero nunca con más de la mayoría absoluta de las preferencias. También muestra que Alejandro Guillier fue su principal rival, hasta la repentina aparición de Beatriz Sánchez en la carrera. A partir de abril de 2017 las encuestas mostraron que, si bien Sebastián Piñera pasaría sin problemas a una segunda vuelta, no era claro si sería junto a Alejandro Guillier o Beatriz Sánchez.
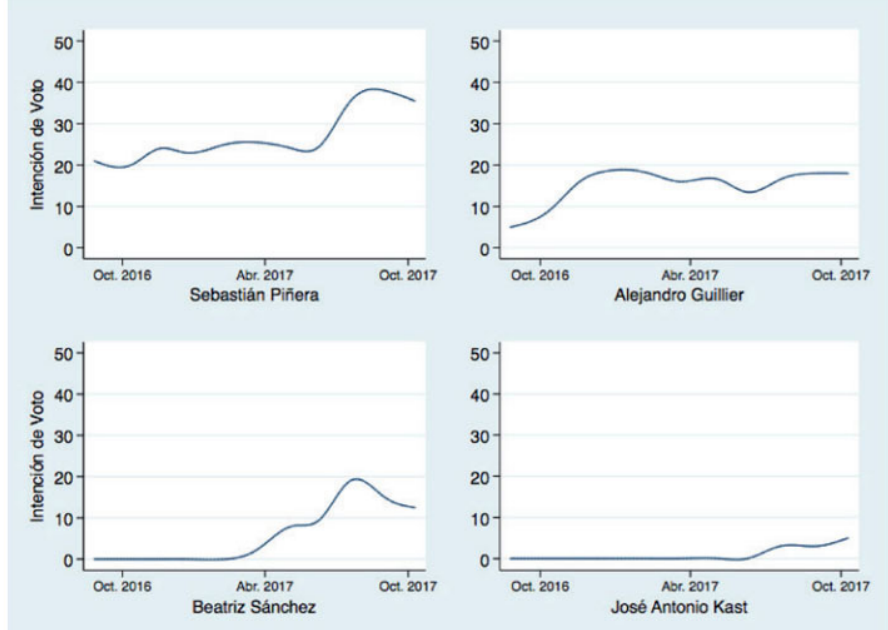

Figura 1. Encuestas de opinión pública

Fuente: Adimark; Cadem; CEP; Criteria Research; Mori-CERC.

Nota: Línea representa mediana cruzada de todas las encuestas publicadas, por candidato.

\section{Resultados de la elección}

La primera vuelta de la elección presidencial se llevó a cabo el 17 de noviembre de $2017^{28}$. La figura 2 muestra los índices de participación electoral en las siete elecciones presidenciales que se han celebrado desde el retorno de la democracia. Muestra que desde entonces el índice de participación electoral ha caído 41 puntos, de $87 \%$ en

28. El mismo día se llevaron a cabo las elecciones legislativas y de consejeros regionales (Cores). En esta elección se presentaron 1382 candidatos para ocupar 278 escaños. Las elecciones se realizaron en el ámbito de circunscripción provincial usando un sistema proporcional. 
1989 a 46\% en 2017. Parte de la caída se debe a la reciente transición del régimen de voto obligatorio a voto voluntario, que se implementó por primera vez en la elección municipal de $2012^{29}$.

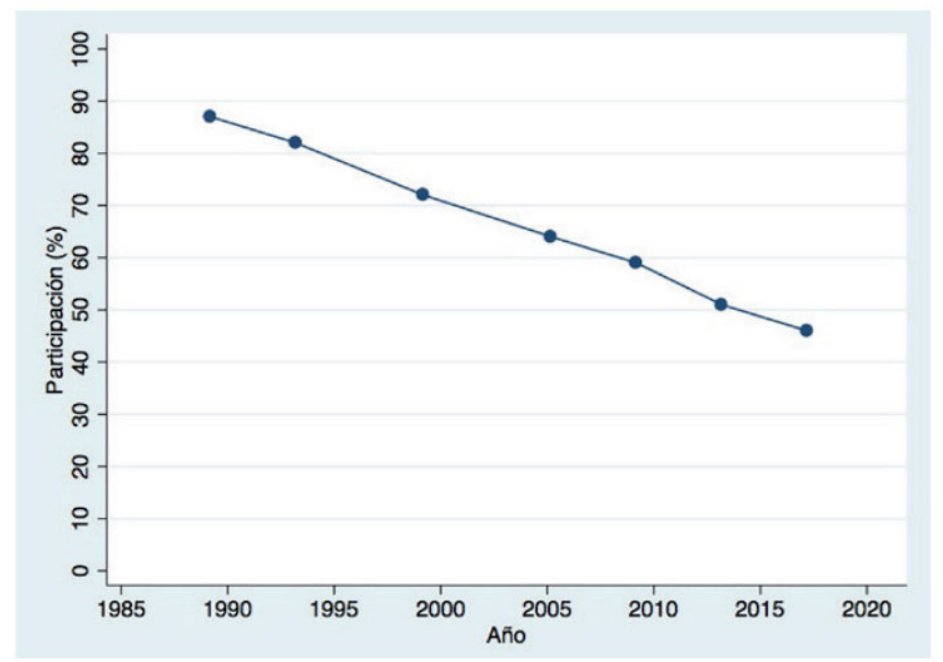

Figura 2. Participación electoral en elecciones presidenciales, 1989-2017.

Fuente: Servicio Electoral de Chile.

La tabla 2 muestra los resultados de la primera vuelta. Muestra que Sebastián Piñera fue el candidato más votado, con $36,6 \%$ de los votos. También muestra que el segundo lugar fue para Alejandro Guillier, y que el tercero fue para Beatriz Sánchez. Por una diferencia de un poco más de cien mil votos, el senador logró pasar a la segunda un mes después. El cuarto lugar fue para José Antonio Kast, quien dejó a Carolina Goic en el quinto lugar y a Marco Enríquez-Ominami en el sexto. Ni Edaurdo Artés ni Alejandro Navarro lograron superar el uno por ciento de los votos.

Lo relevante del resultado de la primera vuelta es que la suma de votos entre los dos candidatos de derecha fue menor a la mayoría absoluta de los votos. Es decir, la suma de los votos de los candidatos de izquierda fue mayor al cincuenta por ciento más uno. Teóricamente, una coordinación de los candidatos de centro e izquierda le hubiera dado la victoria a Alejandro Guillier. Por eso, el gran desafío del senador en la antesala de la segunda vuelta fue tratar de cohesionar a todas las fuerzas progresistas.

Los resultados de la segunda vuelta muestran que no fue posible. Sebastián Piñera logró derrotar a Alejandro Guillier por 54,6\% a 45,4\%. Un análisis más fino de los votos permite inferir que Sebastián Piñera no solo logró mantener sus votos y recoger muchos de los de José Antonio Kast, y algunos de Carolina Goic, sino que, además,

29. Véanse BARNES y RANGEL (2014); CONTRERAS et al. (2016). 
captar la mayoría de los votos de las personas que no participaron en la primera vuelta, pero sí lo hicieron en la segunda (casi cuatrocientas mil) ${ }^{30}$. En esta línea, la incapacidad para articular un frente progresista cohesionado fue funcional para darle la victoria a Sebastián Piñera.

Ganó el candidato con mayor capacidad de coordinación. Esto ya había ocurrido en elecciones anteriores. En 2005, la derecha se dividió en dos grandes candidaturas, pero su incapacidad para coordinarse le dio la victoria a Michelle Bachelet en la segunda vuelta ${ }^{31}$. Asimismo, en 2009, la izquierda se dividió en tres candidaturas, pero su incapacidad para coordinarse le dio la victoria a Sebastián Piñera ${ }^{32}$. Tal como Joaquín Lavín le dio la victoria a Michelle Bachelet en 2005 y Marco Enríquez-Ominami le dio la victoria a Sebastián Piñera en 2009, Beatriz Sánchez le dio la victoria a Sebastián Piñera en 2017.

La descoordinación de los partidos de centroizquierda fue el factor determinante en la definición de la segunda vuelta de 2017. La división de la izquierda en seis candidaturas le permitió a Sebastián Piñera ganar en segunda vuelta. Entre todas ellas, la participación de Beatriz Sánchez fue la más perjudicial para Alejandro Guillier. A pesar de sumar alrededor del $42 \%$ de los votos entre ambos en primera vuelta, la animosidad del periodo de campaña dividió a los votantes de tal manera que fue imposible volver a unificarlos para votar en contra de Sebastián Piñera en la segunda vuelta.

30. La suma de votos por Sebastián Piñera y José Antonio Kast en la primera vuelta fue 2940429. Los votos por Sebastián en la segunda vuelta fueron 3796 579. Es decir, logró captar 856150 votos adicionales entre la primera y segunda vuelta. Dado que solo votaron de forma válida 360152 votantes más en la segunda vuelta, es racional presumir que entraron votos nuevos entre la primera y segunda vuelta que fueron mayoritariamente para Sebastián Piñera. Pero también es racional presumir que muchos de los votantes que votaron por algunos de los seis candidatos restantes en la primera vuelta, específicamente por la candidata ideológicamente más cercana a la derecha (Carolina Goic), prefirieron a Sebastián Piñera por sobre Alejandro Guillier.

31. ANGELL y REIG (2006).

32. BUNKER (2010). 
Tabla 2. Resultados de la elección presidencial 2017.

\begin{tabular}{|c|c|c|c|c|}
\hline & \multicolumn{2}{|c|}{ Primera vuelta } & \multicolumn{2}{|c|}{ Segunda vuelta } \\
\hline & $\begin{array}{l}\text { Número de } \\
\text { votos }\end{array}$ & $\begin{array}{r}\text { Porcentaje de } \\
\text { votos }\end{array}$ & $\begin{array}{l}\text { Número de } \\
\text { votos }\end{array}$ & $\begin{array}{c}\text { Porcentaje de } \\
\text { votos }\end{array}$ \\
\hline Sebastián Piñera & 2.417 .216 & 36.6 & 3.796 .579 & 54.6 \\
\hline Alejandro Guillier & 1.497 .116 & 22.7 & 3.159 .902 & 45.4 \\
\hline Beatriz Sánchez & 1.336 .824 & 20.3 & & \\
\hline José Antonio Kast & 523.213 & 7.9 & & \\
\hline Carolina Goic & 387.780 & 5.9 & & \\
\hline Marco Enríquez-O. & 376.471 & 5.7 & & \\
\hline Eduardo Artés & 33.690 & 0.5 & & \\
\hline Alejandro Navarro & 24.019 & 0.4 & & \\
\hline Total* & 6.596 .329 & 100.0 & 6.956 .481 & 100.0 \\
\hline
\end{tabular}

Fuente: Servicio Electoral de Chile. * Solo suma el total de votos válidos; no suma votos nulos ni blancos.

La tabla 3 muestra los resultados de la elección de senadores de 2017. Muestra una correspondencia entre los dos candidatos presidenciales que pasaron a segunda vuelta y la votación de los senadores de sus respectivas coaliciones. Los candidatos recibieron más o menos los mismos votos que sus listas legislativas. Sin embargo, ambas listas también recibieron un mayor porcentaje de escaños que de votos. Por su parte, Beatriz Sánchez obtuvo el doble de votos que su lista de candidatos senatoriales y Carolina Goic obtuvo la mitad de los votos que la suya.

Considerando que se renovó la mitad del Senado, los doce senadores de Chile Vamos se sumaron a los siete que venían del periodo anterior, para sumar diecinueve, y convertirse en la primera fuerza del Poder Legislativo para el periodo 2018-2022. Los siete senadores de La Fuerza de La Mayoría se sumaron a los ocho que venían del periodo anterior, para sumar quince. Los tres escaños conseguidos por Convergencia Democrática le permitieron convertirse en la tercera fuerza. Por su parte, el senador elegido del Frente Amplio se suma a dos senadores independientes elegidos en 2013. 
Tabla 3.Resultados de la elección de senadores 2017.

\begin{tabular}{|l|c|c|c|c|}
\hline & $\begin{array}{c}\text { Número de } \\
\text { votos }\end{array}$ & $\begin{array}{c}\text { Porcentaje de } \\
\text { votos }\end{array}$ & $\begin{array}{c}\text { Número de } \\
\text { escaños }\end{array}$ & $\begin{array}{c}\text { Porcentaje de } \\
\text { escaños }\end{array}$ \\
\hline Chile Vamos & 628.320 & 37,7 & 12 & 52,2 \\
\hline $\begin{array}{l}\text { La Fuerza de la } \\
\text { Mayoría }\end{array}$ & 380.203 & 22,8 & 7 & 30,4 \\
\hline Frente Amplio & 184.333 & 11,1 & 1 & 4,3 \\
\hline $\begin{array}{l}\text { Convergencia } \\
\text { Democrática }\end{array}$ & 238.644 & 14,3 & 3 & 13,1 \\
\hline Por Todo Chile & 22.929 & 1,4 & 0 & 0 \\
\hline $\begin{array}{l}\text { Coalición Re- } \\
\text { gionalista Verde }\end{array}$ & 2.916 & 0,2 & 0 & 0 \\
\hline Otros* & 208.998 & 12,5 & 0 & 0 \\
\hline Total** & $\mathbf{1 . 6 6 6 . 3 4 3}$ & $\mathbf{1 0 0 . 0}$ & $\mathbf{2 3}$ & $\mathbf{1 0 0 . 0}$ \\
\hline
\end{tabular}

Fuente: Servicio Electoral de Chile. " Incluye independientes y otras coaliciones"*. Solo suma el total de votos válidos; no suma votos nulos ni blancos.

La tabla 4 muestra los resultados de la elección de diputados de 2017. Muestra un patrón similar al de la elección senatorial, al haber una correspondencia entre la votación de los dos principales candidatos. La votación de Chile Vamos se asimila a la proporción de votos recibido por Sebastián Piñera, y la votación de La Fuerza de la Mayoría se asimila a la votación de Alejandro Guillier. Y al igual que en la elección senatorial, estas dos listas fueron las únicas beneficiadas en la traducción de votos a escaños, recibiendo más escaños de lo que les correspondería en un sistema perfectamente proporcional.

Los resultados también muestran una correspondencia con la elección senatorial, en la cual la lista legislativa del Frente Amplio obtuvo menos votos que su candidata presidencial y que la lista legislativa de Convergencia Democrática obtuvo más votos que la suya. La obtención de más de 10\% de los votos para cada una de estas dos terceras fuerzas es un hecho histórico inédito. En todas las elecciones anteriores las dos grandes coaliciones se llevaban más del noventa por ciento de los votos y escaños en las elecciones de diputados. 
Tabla 4.Resultados de la elección de diputados 2017.

\begin{tabular}{|l|c|c|c|c|}
\hline & $\begin{array}{c}\text { Número de } \\
\text { votos }\end{array}$ & $\begin{array}{c}\text { Porcentaje de } \\
\text { votos }\end{array}$ & $\begin{array}{c}\text { Número de } \\
\text { escaños }\end{array}$ & $\begin{array}{c}\text { Porcentaje de } \\
\text { escaños }\end{array}$ \\
\hline Chile Vamos & 2.318 .719 & 38,7 & 72 & 46,5 \\
\hline $\begin{array}{l}\text { La Fuerza de la } \\
\text { Mayoría }\end{array}$ & 1.442 .196 & 24,1 & 43 & 27,7 \\
\hline Frente Amplio & 989.353 & 16,5 & 20 & 12,9 \\
\hline $\begin{array}{l}\text { Convergencia } \\
\text { Democrática }\end{array}$ & 640.612 & 10,6 & 14 & 9,0 \\
\hline Por Todo Chile & 235.576 & 3,9 & 1 & 0,6 \\
\hline $\begin{array}{l}\text { Coalición Re- } \\
\text { gionalista Verde }\end{array}$ & 115.323 & 1,9 & 4 & 2,6 \\
\hline Otros* & 255.471 & 4,3 & 1 & 0,7 \\
\hline Total** & $\mathbf{5 . 9 9 7 . 2 5 0}$ & $\mathbf{1 0 0 , 0}$ & $\mathbf{1 5 5}$ & $\mathbf{1 0 0 , 0}$ \\
\hline
\end{tabular}

Fuente: Servicio Electoral de Chile. * Incluye independientes y otras coaliciones"**. Solo suma el total de votos válidos; no suma votos nulos ni blancos.

\section{Análisis de los resultados}

Lo más relevante de los resultados de la elección presidencial es la importante votación de la candidata de la tercera fuerza, que logró distorsionar el resultado final de la elección. Su votación es testimonio de que no solo hay dos coaliciones dominantes, sino que al menos tres. Si bien la presentación de Beatriz Sánchez en 2017 se parece en alguna medida a la de Marco Enríquez-Ominami en 2009, hay una gran diferencia: el rol de las listas legislativas. La de Beatriz Sánchez obtuvo en su primera elección más de veinte veces lo que obtuvo la lista de Marco Enríquez Ominami en la suya.

En esa línea, los resultados de las elecciones legislativas muestran un fuerte quiebre en el ordenamiento del sistema de partidos. La entrada de una tercera y cuarta fuerza al Poder Legislativo cambia la composición del Congreso, y como tal la naturaleza de las relaciones entre los partidos, al igual que la relación entre el gobierno y la oposición. La distribución de poder dicotómica que caracterizó el sistema de partidos desde 1990 se acabó en 2018. La entrada de las nuevas fuerzas es evidencia del quiebre.

Este fraccionamiento se puede observar en el aumento en el número de partidos que compite en elecciones y que obtiene representación legislativa. La figura 3 muestra la evolución del número efectivo de partidos electorales (NEPE) y legislativos (NEPL), de acuerdo con el índice de Markuu Laakso y Rein Taagepera ${ }^{33}$. Muestra que en la elección de 2017 el NEPE y el NEPL llegaron en su punto más alto desde 1949. 
Muestra que por primera vez en más de medio siglo hay más de diez partidos relevantes compitiendo en elecciones, y más de siete representados en el Congreso.

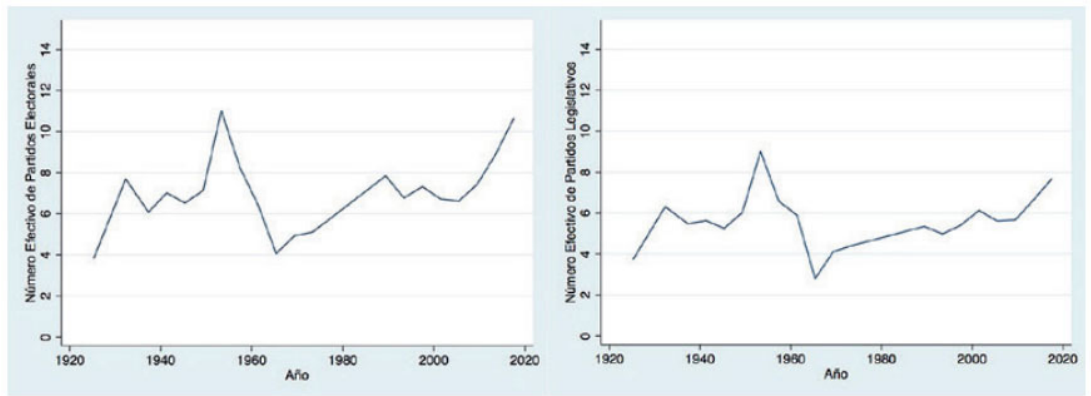

Figura 3. Número efectivo de partidos, 1925-2018

Fuente: Servicio Electoral de Chile.

Nota: No hubo elecciones entre 1973 y 1989.

El fraccionamiento del sistema de partidos es claramente visible en los resultados de la elección de diputados, donde cuatro grandes coaliciones obtuvieron más del $10 \%$ de los votos. También es visible en el alto porcentaje de escaños que cada una de esas coaliciones obtuvo. Los resultados no solo muestran un quiebre con los patrones, sino, además, con la tendencia histórica. El fraccionamiento del sistema de partidos, que se da a partir de la elección de 2017, es incluso superior al que hubo en los años previos al quiebre de la democracia en $1973^{34}$.

Un dato importante que ayuda a caracterizar este quiebre está en los índices de reelección, que en este caso sugieren que junto con la modificación en la estructura del sistema de partidos hay una renovación significativa en la composición de los legisladores. Los datos sugieren que la elección de 2017 no solo fue la instancia en que menos senadores y diputados buscaron ser reelectos, sino que también la instancia en que menos de ellos la consiguieron. En otras palabras, fue la elección de mayor recambio legislativo desde el retorno de la democracia.

La tabla 5 muestra las tasas de reelección en el Senado desde 1989. Como se adelanta más atrás, muestra que, en comparación con todas las elecciones anteriores, la de 2017 fue la instancia en la que menos senadores buscaron la reelección (junto con 1997 y 2013) y en que menos senadores la consiguieron (junto con 2013). Presumiblemente, esto está relacionado con los escándalos de corrupción, pues aquello explicaría porqué varios de los titulares no fueron a la reelección (como Jaime Orpis), y porque varios de los que fueron, perdieron (como Fulvio Rossi).

34. Véase VALENZUELA (1978). 
Tabla 5. Reelección en el Senado, 1993-2017

\begin{tabular}{|l|c|c|c|c|c|}
\hline \multirow{2}{*}{ Año } & \multicolumn{2}{|c|}{$\begin{array}{c}\text { Senadores que buscaron la } \\
\text { reelección }\end{array}$} & $\begin{array}{c}\text { Senadores que consiguieron } \\
\text { la reelección }\end{array}$ & $\begin{array}{c}\text { Total de es- } \\
\text { caños }\end{array}$ \\
\cline { 2 - 6 } & Número & Porcentaje & Número & Porcentaje & Número \\
\hline 1993 & 15 & 83,3 & 9 & 60,0 & 18 \\
\hline 1997 & 10 & 50,0 & 7 & 70,0 & 20 \\
\hline 2001 & 11 & 61,1 & 9 & 81,8 & 18 \\
\hline 2005 & 12 & 60,0 & 7 & 58,3 & 20 \\
\hline 2009 & 11 & 61,1 & 7 & 63,6 & 18 \\
\hline 2013 & 10 & 50,0 & 6 & 60,0 & 20 \\
\hline 2017 & 10 & 43,4 & 6 & $60 ; 0$ & 23 \\
\hline Total & $\mathbf{7 9}$ & $\mathbf{5 8 , 4}$ & $\mathbf{5 1}$ & $\mathbf{6 4 , 8}$ & $\mathbf{1 3 7}$ \\
\hline
\end{tabular}

Fuente: Servicio Electoral de Chile.

La tabla 6 muestra las tasas de reelección en la Cámara de Diputados. En términos comparativos, la elección de 2017 no solo fue la elección con la menor cantidad de diputados a la reelección (66,6\%), sino que, también, fue la elección con la menor cantidad de diputados reelectos $(76,2 \%)$. Tomando en cuenta los datos de reelección para el Senado, estas cifran posicionan a la elección legislativa de 2017 como instancia en que han entrado más legisladores (senadores y diputados) novatos desde el retorno de la democracia.

Tabla 6. Reelección en la Cámara de Diputados, 1993-2017.

\begin{tabular}{|l|c|c|c|c|c|}
\hline \multirow{2}{*}{ Año } & \multicolumn{2}{|c|}{$\begin{array}{c}\text { Diputados que buscaron la } \\
\text { reelección }\end{array}$} & $\begin{array}{c}\text { Diputados que consiguieron } \\
\text { la reelección }\end{array}$ & $\begin{array}{c}\text { Total de es- } \\
\text { caños }\end{array}$ \\
\cline { 2 - 6 } & Número & Porcentaje & Número & Porcentaje & Número \\
\hline 1993 & 87 & 72,5 & 70 & 80,5 & 120 \\
\hline 1997 & 86 & 71,6 & 73 & $84 ; 9$ & 120 \\
\hline 2001 & 92 & 76,6 & 72 & 78,3 & 120 \\
\hline 2005 & 92 & 76,6 & 77 & 83,7 & 120 \\
\hline 2009 & 91 & 75,8 & 74 & 81,3 & 120 \\
\hline 2013 & 91 & 75,8 & 77 & 84,6 & 120 \\
\hline 2017 & 80 & 66,6 & 61 & 76,2 & 155 \\
\hline Total & $\mathbf{6 1 9}$ & $\mathbf{7 3 , 6}$ & $\mathbf{5 0 4}$ & $\mathbf{8 1 , 3}$ & $\mathbf{8 7 5}$ \\
\hline
\end{tabular}

Fuente: Servicio Electoral de Chile. 


\section{Escenario político resultante}

La elección presidencial y legislativa de 2017 es relevante en dos maneras. Primero, porque marca un cambio en la dirección política del país. El retorno de Sebastián Piñera al poder prueba que el modelo progresista de izquierda por Michelle Bachelet no fue bien recibido por la mayoría de los chilenos. Al constituirse como un referéndum en cuanto a la administración de la Presidenta, la elección se puede interpretar como una donde los chilenos privilegiaron la bonanza económica por sobre la reforma estructural.

La elección también es relevante porque marcó el inicio de un nuevo sistema de partidos. El ordenamiento sobre la base de dos grandes coaliciones fue reemplazado por uno de tres o cuatro coaliciones diferentes. Esto tiene algunos efectos notorios. A diferencia del ordenamiento anterior, donde el gobierno de turno tomaba las decisiones de forma unilateral, el nuevo ordenamiento obliga a involucrar a la oposición. A pesar de ser un sistema más fraccionado, es uno más fluido también.

Cabe destacar que la legislatura que debutó en el periodo 2018-2022 es la con más miembros debutantes desde el retorno de la democracia ${ }^{35}$. Esto es evidencia adicional de que el sistema cambió. Los partidos antiguos perdieron escaños a costa de los nuevos. Mientras que la renovación de poder en la izquierda se dio en un espacio de reemplazo (RD como suplemento del PS), en la derecha se dio en uno de renovación (Evopoli como complemento de la UDI).

Una pregunta relevante que nace de lo anterior es cómo este nuevo escenario afecta la gobernabilidad. Algunos han sostenido que fragmentaciones en el sistema de partidos naturalmente conducen a menor capacidad de gobernabilidad y, a veces, a crisis, como en Chile en el periodo pre $1973^{36}$. La lógica es que jefes de gobierno con apoyo minoritario en presidencialismo tienen problemas estructurales para legislar ${ }^{37}$. Sin embargo, hay quienes encuentran lo contrario ${ }^{38}$, pues sugieren que la fragmentación puede conducir a más oportunidades de negociación.

De hecho, hay varios elementos en el nuevo contexto multipartidista de Chile que sugieren que la segunda línea es más aplicable, a partir de 2018. Pues, hay razones para creer que el gobierno elegido para el periodo 2018-2022 tiene menos posibilidades de estancarse legislativamente que los anteriores. Si el bloque tradicional de centroizquierda no quiere cooperar con Sebastián Piñera, el Presidente puede optar por negociar con el nuevo bloque de centroizquierda o, incluso, con la reducida, pero disciplinada bancada de la Democracia Cristiana.

35. Véanse BUNKER y NAVIA (2015).

36. VALENZUELA (1978).

37. LINZ (1990).

38. CHEIBUB (2007). 
Los primeros pasos del gobierno debutante en 2018 sugieren aquello. Al no tener los votos para legislar unilateralmente, optó por un método basado en negociaciones y pactos multilaterales. En vez de arriesgar caer en parálisis legislativa por no tener el apoyo suficiente, buscó construir puentes con partidos de todos los colores políticos. Sin estar ideológicamente alineado, buscó abrir líneas de conversaciones abiertas y simultáneas con todos los partidos.

Esto fue lo que ocurrió, por ejemplo, con la conformación de las mesas de trabajo de Seguridad, Infancia, Araucanía, Salud y Desarrollo, a las cuales asistieron desde conocidos exministros a políticos emergentes de izquierda ${ }^{39}$. Todo indica que el nuevo modelo a seguir es el ecuatoriano, de coaliciones fantasmas, donde distintos proyectos de ley se diseñan, negocian y aprueban con el apoyo de distintos sectores ${ }^{40}$. Al contrario de lo que se ha sostenido en el pasado, mayor fragmentación implica mayor flexibilidad, y en algunos casos, incluso, mayor gobernabilidad.

\section{Referencias bibliográficas}

ALEMÁN, Eduardo (2008). "Policy positions in the chilean senate: An analysis of coauthorship and roll call data". Brazilian Political Science Review, 2(2): 74-92.

ANGELL, Alan y Cristóbal REIG (2006). "Change or continuity? The Chilean elections of 2005/2006". Bulletin of Latin American Research 25(4): 481-502.

ARANA, Ignacio (2017). “Chile 2016: ¿El nadir de la legitimidad democrática?”. Revista de Ciencia Política, 37(2): 305-334.

BARNES, Tiffany D., y Gabriela RANGEL (2014). "Subnational patterns of participation: Compulsory voting and the conditional impact of institutional design". Political Research Quarterly, 71(4): 826-841.

BIOBIO (2017). "Eduardo Artés, candidato presidencial: Corea del Norte es una democracia popular." Disponible en www.biobiochile.cl/noticias/nacional/regiondel-bio-bio/2017/o8/24/eduardo-artes-candidato-presidencial-corea-del-nortees-una-democracia-popular.shtml [Fecha de consulta: 14 de noviembre de 2018].

BUNKER, Kenneth (2010). The 2009 Presidential Election of Chile. San Diego, CA: San Diego State University.

BUNKER, Kenneth, y Patricio NAVIA (2015). "Incumbency Advantage and Tenure Length in the Chilean Chamber of Deputies, 1989-2009". Revista de Ciencia Politica, 35(2): 251-271.

BUNKER, Kenneth. "El éxodo electoral de la DC.” Disponible en www.tresquintos. $\mathrm{cl} / 2017 / 05 / 09 /$ el-exodo-electoral-de-la-dc/ [Fecha de consulta: 14 de noviembre de 2018].

39. La Tercera (2018).

40. Véase MEJÍA ACOSTA (2006). 
BUNKER, Kenneth (2018). "Retiros, Remociones y Renuncias en el Poder Legislativo de Chile: 1990-2018". Documentos de Trabajo Universidad Central.

CHEIBUB, José Antonio (2007). Presidentialism, parliamentarism, and democracy. Cambridge: Cambridge University Press.

CONTRERAS, Gonzalo, Alfredo JOIGNANT, y Mauricio MORALES (2015)“'The return of censitary suffrage? The effects of automatic voter registration and voluntary voting in Chile". Democratization, 23(3): 520-544.

COOPERATIVA (2017). "A dos días de la elección, dos expertos electorales revelan sus pronósticos”. Disponible en www.cooperativa.cl/noticias/pais/politica/ presidenciales/a-dos-dias-de-la-eleccion-dos-expertos-electorales-revelansus/2017-11-17/091026.html/ [Fecha de consulta: 14 de noviembre de 2018].

COX, Gary W (1997). Making votes count: Strategic coordination in the world's electoral systems. New York: Cambridge University Press.

El Mercurio (2017). "Felipe Larraín: 'La economía chilena requiere una cirugía mayor"'.Disponible en www.elmercurio.com/Inversiones/Noticias/FondosMutuos/2017/o1/o3/Felipe-Larrain-La-economa-chilena-requiere-una-cirugiamayor.aspx [Fecha de consulta: 14 de noviembre de 2018].

FÁBREGA, Jorge, Jorge GONZÁLEZ, y Jaime LINDH (2018). "Polarization and Electoral Incentives: The End of the Chilean Consensus Democracy, 1990-2014". Latin American Politics and Society, 6o(4): 49-68.

FUENTES, Claudio (2018). "Debate constitucional en Chile ¿Reemplazo vía enmienda?". Política y Gobierno, 25(2): 469-483.

GAMBOA, Ricardo y Mauricio MORALES (2016). "Chile's 2015 Electoral Reform: Changing the Rules of the Game". Latin American Politics and Society, 58(4): 126-14.4.

GAMBOA, Ricardo y Carolina SEGOVIA (2016). "Chile 2015: Falla política, desconfianza y reforma”. Revista de Ciencia Política, 36(1): 123-144.

LAAKSO, Markuu y Rein TAAGEPERA (1979). "Effective number of parties: A measure with application to West Europe". Comparative Political Studies, 12(1):3-27.

La Nación (2017). “Grieta: Chile se asoma a una polarización inédita por un ballottage de final incierto". Disponible en www.lanacion.com.ar/2092144grieta-chile-seasoma-a-una-polarizacion-inedita-por-un-ballottage-de-finalincierto. [Fecha de consulta: 14 de noviembre de 2018].

La Tercera (2017). "Caso OAS: fiscal Chong alista segundo viaje a Brasil para interrogar a Duda Mendonça.” Disponible en www2.latercera.com/noticia/ caso-oasfiscal-chong-alista-segundo-viaje-brasil-interrogar-duda-mendonca [Fecha de consulta: 14 de noviembre de 2018]. 
La Tercera (2018). "Gobierno creará nuevas mesas de trabajo para llegar a acuerdos." Disponible en www.latercera.com/politica/noticia/gobierno-creara-nuevas-mesastrabajo-llegar-acuerdos/249707/ [Fecha de consulta: 14 de noviembre de 2018].

LINZ, Juan J. (1990). "The perils of presidentialism”. Journal of Democracy, 1(1): 51-69.

LONDREGAN, John B.(20oo). Legislative institutions and ideology in Chile. New York: Cambridge University Press.

JONES, Mark P. (1999). "Electoral laws and the effective number of candidates in presidential elections". The Journal of Politics, 61(1):171-84.

MEJÍA ACOSTA, Andrés (2006). "Crafting legislative ghost coalitions in Ecuador: Informal institutions and economic reform in an unlikely case". En Informal Institutions and Democracy: Lessons from Latin America (Baltimore, Johns Hopkins University Press), pp. 69-84.

MORALES, Mauricio (2017). "Experto electoral DC proyecta debacle parlamentaria si partido opta por lista separada". Disponible en: www.t13.cl/noticia/politica/ experto-dc-proyecta-debacle-parlamentaria-si-partido-opta-camino-propio [Fecha de consulta: 14 de noviembre de 2018].

NAVIA, Patricio (2005). "La transformación de votos en escaños: Leyes electorales en Chile 1833-2004". Política y Gobierno, 12(2): 233-276.

NAVIA, Patricio (2018). "¿Si puedes repararla, para qué reemplazarla? Democratizar la Constitución de Pinochet en Chile", Política y Gobierno, 25(2): 485-499.

NETO, Octavio Amorim y Gary W. COX (1997). "Electoral institutions, cleavage structures, and the number of parties". American Journal of Political Science, 41(1): 149-174.

RAE, DOUGLAS W. (1967). The Political Consequences of Electoral Laws. New Haven y Landon: Yale University Press.

SHUGART, Matthew S., y Rein TAAGEPERA (2017). Votes from Seats: Logical Models of Electoral Systems. New York: Cambridge University Press.

TELETRECE (2016): "La paulatina partida del gabinete más íntimo de Bachelet”. Disponible en www.t13.cl/noticia/politica/la-paulatina-partida-del-gabinetemas-intimo-bachelet/ [Fecha de consulta: 14 de noviembre de 2018].

TORO, Sergio y VALENZUELA, Macarena (2018): "Chile 2017: Ambiciones, estrate- gias y expectativas en el estreno de las nuevas reglas electorales". Revista de Ciencia Política, 38(2): 207-232.

VALENZUELA, Arturo (1978): The breakdown of democratic regimes, Chile (Baltimore, Johns Hopkins University Press). 